\title{
Simulation analysis of bubble motion under ultrasonic assisted Electrical Discharge Machining
}

\author{
Wenjun Kong ${ }^{a}$, Ce Guo ${ }^{b}$ and Xijing Zhu ${ }^{\mathrm{c}}$ \\ School of Mechanical and Power Engineering, North University of China, Taiyuan 030051, China. \\ akwj2002@163.com, bouoce1027@163.com, 'ZXJ161501@nuc.edu.cn
}

Keywords: ultrasonic, bubble, electrical discharge machining, FLUENT.

\begin{abstract}
In order to reveal the machining mechanism of the inter electrode gap during ultrasonic assisted electrical discharge machining, the relationship between the distribution of the flow field and bubble motion was studied. The three-dimensional model of deep hole machining was established firstly using the GAMBIT software. On the basis of theoretical analysis on the gap of the flow field working liquid, the bubble motion in the process of multiple pulse discharge was simulated using the FLUENT software. The motion of a single bubble and bubbles under with and without ultrasound was simulated respectively. Compared with the simulation results without considering ultrasound, it can be obtained that ultrasonic vibration is helpful for the generation of bubbles and improvement in the machining efficiency. It is provided a simulation basis for the actual analysis of electrical discharge machining process and the optimization of machining parameters.
\end{abstract}

\section{Introduction}

Electrical Discharge Machining (EDM) has been widely used in manufacturing molds, aerospace, automotive and aviation medicine [1-2]. Ultrasonic assisted EDM is a compound machining technology, which ultrasonic vibration is added tool electrode or workpiece electrode on the basis of EDM to improve the machining conditions. It is due to the fact that the gap of EDM is in the range of micro-scale and general processing material is opaque, the measurement of the flow field by putting measuring instrument into the gap is almost impossible. On the other hand, the movement of the flow field of EDM gap is so complex that it is difficult to obtain the analytical solution directly by theoretical calculations. Moreover, the bubble volume generated by the inter electrode gap in the process of EDM is effected differently by some parameters, such as the electrode discharge voltage, discharge current, pulse width, pulse interval, electrode machining time and the conditions of liquid[3-4]. Those parameters make EDM exhibit various stability and efficiency. In the current production practice, the various parameters of EDM are often determined by technicians from experience. However, the technology of finite element simulation provides an effective way to analyze the flow field of EDM gap. Therefore, in this paper, the generation and motion of air bubbles have been studied using FLUENT software, which from the perspective of computer simulation provides a strong guarantee for the actual analysis of the ultrasonic assisted EDM process and optimization of processing parameters.

\section{Theoretical model}

Geometric modeling. The inter electrode gap during ultrasonic assisted EDM is composed of the electrode and the workpiece, and the internal gap is full of the processing liquid. In addition, many chips and bubbles during processing can be produced. Bubbles are majorly made up of hydrocarbon gas and oxygen. The generation time of bubbles is very short, and the bubbles also start doing the movement of contraction and expansion, which can make other working fluid flow. In the whole process, the electric field between poles start to decrease, and bubble motion restores balance after a discharge. After a period of discharge pulse interval, the next pulse discharge begins, and bubbles do contraction and expansion movement repeatedly. In the process of continuous discharge, the simulation of the flow field in the inter electrode gap not only relies on the basic equations of fluid 
dynamics, but also needs to establish the mathematical model of pulse discharge bubble and solve the problem of flow deformation.

The model of the inter electrode gap is simplified as shown in Fig. 1, and then the grid is meshed with GAMBIT software. Afterwards, boundary conditions of ultrasonic assisted EDM were applied as shown in Fig.2.

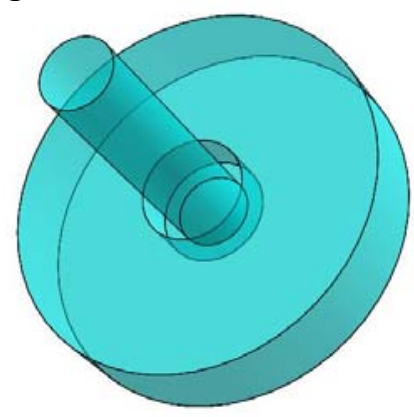

Fig.1 Inter electrode gap of Ultrasonic EDM

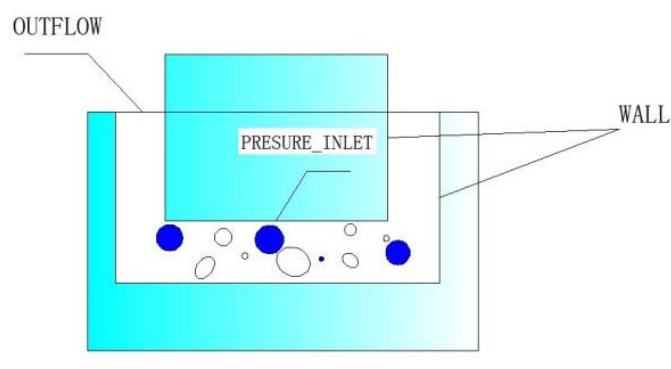

Fig.2 Boundary conditions of Ultrasonic EDM

Mathematical model. The simulation describing the conversions of the processing oil and bubbles is gained by using FLUENT software and VOF model. The volume continuity equation may be as follows:

$$
\frac{\partial}{\partial t}\left(\alpha_{q} \rho_{q}\right)+\nabla\left(\alpha_{q} \rho_{q} v_{q}\right)=\sum_{p=1}^{n}\left(m_{p q}-m_{q p}\right)
$$

where $\rho_{q}$ is q phase density, $v_{\mathrm{a}}$ is the velocity vector of $q$ phase, $m_{p q}$ is the fluid quality from $p$ phase to $q$ phase, $m_{q p}$ is the fluid quality from $q$ phase to $p$ phase.

The user-defined function UDF DEFINE_MASS_TRANSFER can be used to describe the process of processing oil turning into the bubble. The processing oil starts being turned into bubbles as soon as EDM machining is ready to begin. The return value is the conversion rate defined by DEFINE_MASS_TRANSFER and the conversion quality is gotten by the conversion time and the conversion volume.

Processing oil volume fraction is defined by the following equation:

$$
a_{0}+a_{g}=1
$$

where $a_{0}$ is the volume fraction of the processing oil, $a_{\mathrm{g}}$ is the volume fraction of the bubbles.

For the VOF model, a single momentum conservation equation of the whole flow field was solved by the Eq. (3).

$$
\frac{\partial}{\partial t}\left(\rho_{\alpha} v\right)+\nabla\left(\rho_{\alpha} v v\right)=-\nabla p+\nabla\left[\mu_{a}\left(\nabla v+\nabla v^{T}\right)\right]+\rho_{\alpha}+F
$$

where, $\rho$ and $\mu$ are all taken the average of the volume fraction of each phase. If there are existed two phases, the calculation formulas of $\rho_{a}$ and $\mu_{a}$ from Eq. (3) are as follows:

$$
\begin{aligned}
& \rho_{\alpha}=a_{1} \rho_{1}+a_{2} \rho_{2} \\
& \mu_{\alpha}=a_{1} \mu_{1}+a_{2} \mu_{2}
\end{aligned}
$$

where, $a_{1}$ and $a_{2}$ is the volume fraction of two phases respectively.

Numerical method.To obtain the effect of ultrasound on the generation and motion of air bubbles, the flow field of inter electrode gap before and after applying the ultrasound were compared. The simulation was completed by FLUENT simulation. The same calculation conditions were determined when with and without considering ultrasound. In addition, the ultrasonic wave is applied at the bottom of a thin wall cylinder under sine motion conditions by using UDF user-defined functions DEFINE_GRID_MOTION, and the dynamic grid technique is used to implement the whole simulations. 


\section{Results and discussions}

In Fig. 3, the flow field is the phase cloud at the moment of $200 \mu \mathrm{s}$, and the clearance in the underside from the clearance export is neglected. In the phase diagram, the blue part represents EDM oil; the light green part represents the conversion area; the red part represents gas. It can be seen that the variation of red area becomes more and more obvious, which represents the volume of a single bubble becomes greater. The phenomenon demonstrates also that the introduction of ultrasound speeds up the processing of oil liquid to change rapidly, leads to the rate of the generation of air bubbles and enhances the efficiency of gasification. In actual processing, the role of ultrasound also includes the transfer of ultrasonic energy to process oil. What is more, the process of transmission is similar to that of heating effect, which can produce more bubbles. Thus, the effect of ultrasound on single bubble increases the production rate of single bubble visibly.

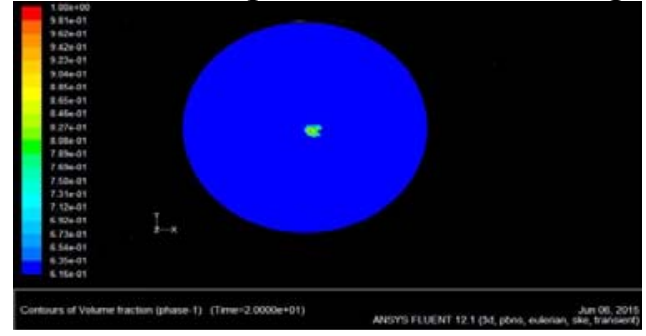

(a) Without considering ultrasonic wave

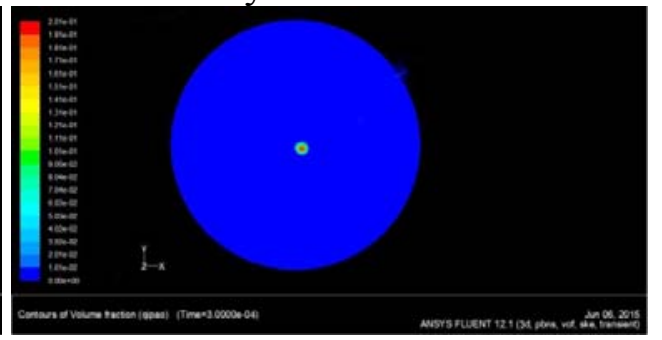

(b) With considering ultrasonic wave

Fig.3 Single bubble distribution under underside of the electrode $(200 \mu \mathrm{s})$

Fig. 4 gives the phase cloud of overlooking the underside from the export of the side gap is the bubble cloud in $5000 \mu \mathrm{s}$ with and without considering ultrasound. From the Fig. 4, the number of bubbles increases obviously by the comparison, and the phenomenon of bubble polymerization even occurs in the process of comparison. But it is worth of attention that the radius of the bubble becomes smaller. The reason of the increasing number of bubbles is the same as that of a single bubble. The joining of ultrasound can reduce the polymerization time of air bubbles and make more bubble nucleus turned into bubbles by reducing the threshold of air bubbles. And the reason for the reduction of the bubble radius is that $5000 \mu \mathrm{s}$ is the end of a cycle of the ultrasound, and there exists the alternating cycle of positive and negative pressure in the process of ultrasonic wave propagation. In the negative phase, the medium molecular is squeezed, and the density of the original medium is increasing; compressing air bubbles can make the radius decrease. In order to describe perfectly the effect of the joining ultrasound on air bubbles on the motion from the bottom gap to the side gap, the bubbles distribution under different positions of the electrode was simulated in Fig. 5.

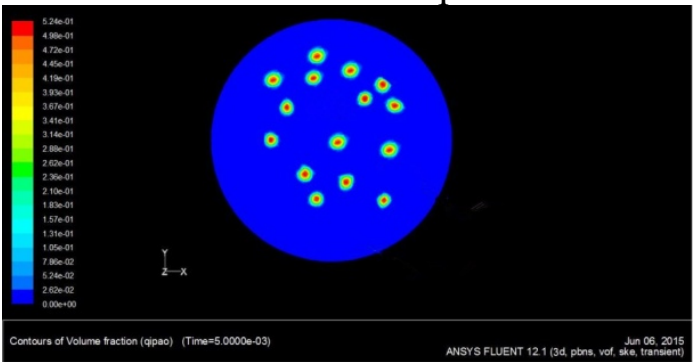

(a) Without considering ultrasonic wave

Fig.4 Cloud chart of bubbles distribution

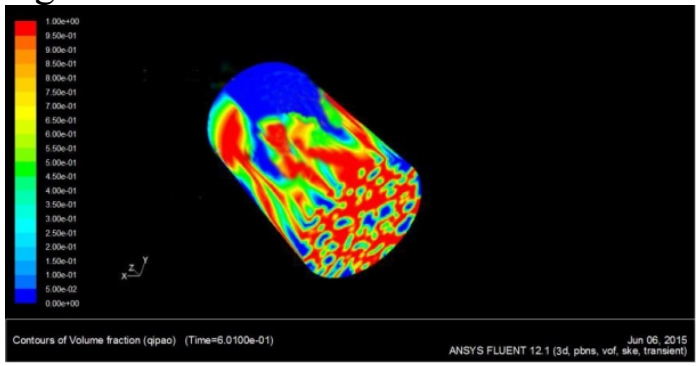

(a) Without considering ultrasonic wave

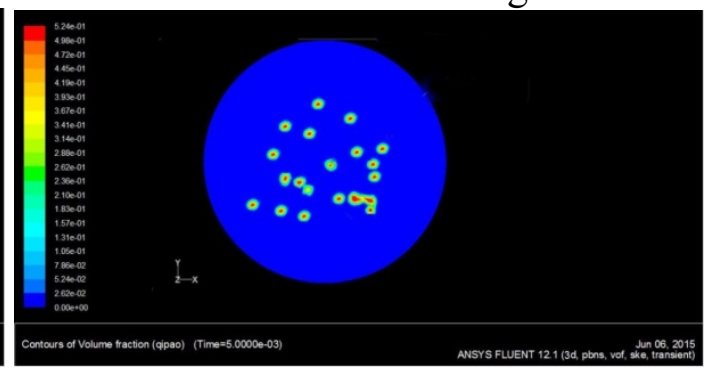

(b) With considering ultrasonic wave under underside of the electrode $(5000 \mu \mathrm{s})$

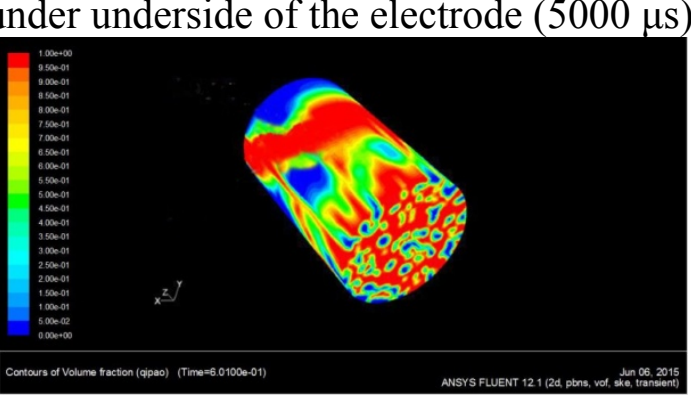

(b) With considering ultrasonic wave

Fig.5 Cloud chart of bubbles distribution under lateral and underside of the electrode $(601 \mathrm{~ms})$ 
From the Fig. 5, bubble cloud was viewed from the lateral and underside of the electrode at $601 \mathrm{~ms}$, in which the red area is for the bubble; the blue area is for processing oil. By the comparison of the two figures, it can be found that the number of bubbles and the bubble fusion rate are all increasing in the time of multi-pulse discharge. Moreover, the increasing number of bubbles shows the intensity of EDM is increasing. Loaded ultrasonic vibration can improve well the clearance status and the processing efficiency.

The reason that the machining efficiency of ultrasonic assisted EDM is higher than that of EDM can be explained as follows:

(1) Because the ultrasound speeds up the changing rate of the workpiece displacement, makes the processing point of discharge change, increase changing of the discharge gap, thus the arc discharge is reduced. And ultrasonic vibration can make electric corrosion product distribute more evenly between the two electrodes in order to help the next discharge point discharge.

(2) Ultrasound also has vibration effect on electric corrosion products like the processing chip. And it is easier to link with the media so as to form a certain discharge channel to increase the discharge rate.

(3) Ultrasonic vibration may cause multi-channel pulse discharge, and improve the number of discharge pulse power. Thus it can improve the utilization rate of discharge to enhance pulse discharge, make the cavitation number increase and the cavitation efficiency improve. And the simulation results of the ultrasound on the producing of bubbles are consistent with theoretical analyses of bubbles from the ultrasound.

\section{Conclusion}

Based on the related theory of EDM, the variations of the bubble volume in the inter electrode gap during ultrasonic assisted EDM were studied using FLUENT software. In the first place, the three-dimensional geometrical modeling of the flow field in the inter electrode gap was established. According to the influential law of the actual discharge on the flow field in the gap, the rate of conversion of the area was determined by the mass transfer function. The ultrasonic vibration was imposed using the dynamic grid technology. The motion of a single bubble and bubble with and without ultrasound was compared respectively. It is concluded that the number of bubbles is increased with considering ultrasound. In addition, ultrasound improves effectively the machining efficiency, and the simulation results are consistent with theoretical analyses. It provides a powerful guarantee for analyzing the actual holes machining process and the optimization of process parameters.

\section{Acknowledgements}

The work was supported by the National Natural Science Foundation of China (51275490 and 50975265) and the Shanxi Province Natural Science Foundation (2013011024-5)

\section{References}

[1] B. M. Schumacher, About the role of debris in the gap during electrical discharge machining, CIRP Ann. Manuf. Technol, 39 (1990) 197-199.

[2] S. Hayakawa, Y. Sasaki, F. Ltoigawa, T. Nakamura, Relationship between occurrence of material removal and bubble expansion in electrical discharge machining, Procedia CIRP, 6 (2013) 174-479.

[3] J. Wang, F. Han, G. Cheng, F. Zhao, Debris and bubble movements during electrical discharge machining, Int J Mach Tools Manuf, 58 (2012) 11-18.

[4] J. C.Su, J. Y. Kao, Y. S. Tang, Optimisation of the electrical discharge machining process using a GA-based neural network, Int J Adv Manuf Technol, 24 (2004) 81-90. 